\title{
Quantitative Description of Land Terrain Correction Factors for Tactical Wireless Communication
}

\author{
Tian Lan a, Ding Yuan, Houde Quan, Peizhang Cui, Huixian Sun, \\ Zhiqiang Tang and Yaobei Wang \\ The Army Engineering University of PLA, Shijiazhuang 050003, China \\ a517851983@qq.com
}

\begin{abstract}
In order to correct the middle value of VHF propagation loss, plan tactical wireless communication network well and guarantee quality of communication, this article firstly contrasts applicability of deterministic models and statistical models in background of tactical wireless communication. Then, for typical land terrain - hilly terrain, slant terrain and edge peaks, the corresponding Okumura correction factors are briefly explained. And it concludes that Okumura correction factors cannot be directly used to correct VHF propagation loss. Finally, according to characteristics of Okumura correction factors, the engineering practice process is reworked out and correction factors are fitted again. Those steps have reference significance for optimizing and extending of Okumura correction factors.
\end{abstract}

Keywords: hilly, peak, slant terrain, correction, optimization.

\section{Introduction}

Radio propagation loss models can be divided into deterministic models and statistical models. Because a deterministic model requires a complete and comprehensive geographical environment conditions and antennas are randomly changed in the mobile environment. For mobile communication, the use of statistical models in mobile communications is appropriate. A statistical model is generally an empirical or semi-empirical formula fitted by a large number of experimental data. The prediction results are statistical inference and have a certain degree of reliability $[1,2]$.

In the practical application of tactical wireless communication, fixed antenna gain, transmission power $(10 \mathrm{~W})$, working band (less than $100 \mathrm{MHz}$ ), and antenna surface height (less than $6 \mathrm{~m}$ ) lead to some problems, that many existing propagation models and corresponding correction factors which are not suitable for tactical wireless communication [3].

If some relatively accurate statistical models can be corrected, that are calibrated by local representative experimental data, they may have higher prediction accuracy and better reliability [4]. The purpose of this paper is to make use of measured data to make Okumura correction factors suitable for tactical wireless communication, improving prediction accuracy of radio propagation loss, fully considering influence of geographic environment on wireless communication planning. Then by increasing antennas power in time and so on, it will be ensuring quality of VHF communication.

\section{Okumura Correction Factors}

Okumura divides typical terrain into hilly land, edge peaks and slant terrain. He uses measured data to fit image and algorithm to correct radio propagation loss on typical terrain, so that the median value of the radio propagation loss is more accurate.

Based on measured data, according to analyzing Okumura correction factor, it can be found that the correction factor of hilly land is closely related to terrain irregularity, frequency, distance and antenna height, the correction factor of an edge peak is closely related to frequency and distance and the correction factor of slant terrain is closely related to the mean inclination, distance and frequency [5]. However, Okumura correction factors are not suited to the tactical wireless communication. For example, frequency of tactical wireless communication is lower. So, we can recalculate and optimize those correction factors by research results of Okumura, so that it can still achieve a certain prediction effect in VHF tactical wireless communication. 


\section{Optimization Methods of Okumura Correction Factors}

Combining with analysis of second section and known conclusions, optimization methods of Okumura correction factors are shown as Fig.1, Fig.2 and Fig.3:

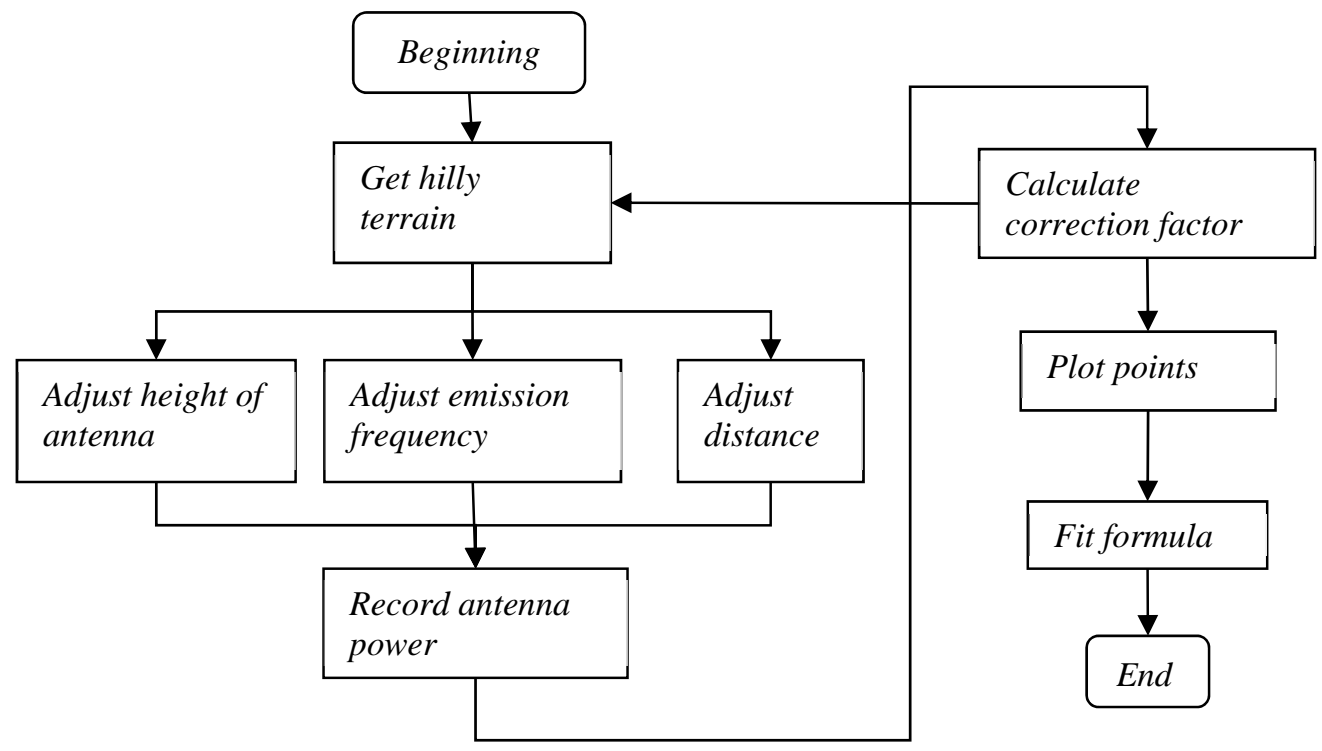

Fig 1. Correction methods for hilly terrain

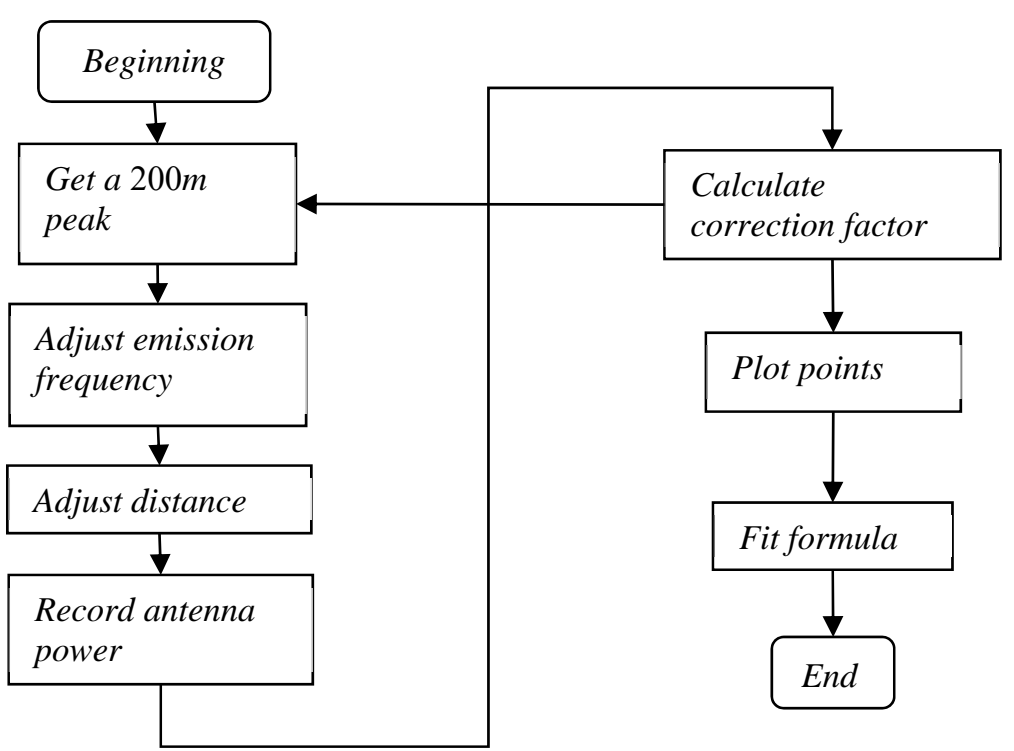

Fig 2. Correction methods for a peak 


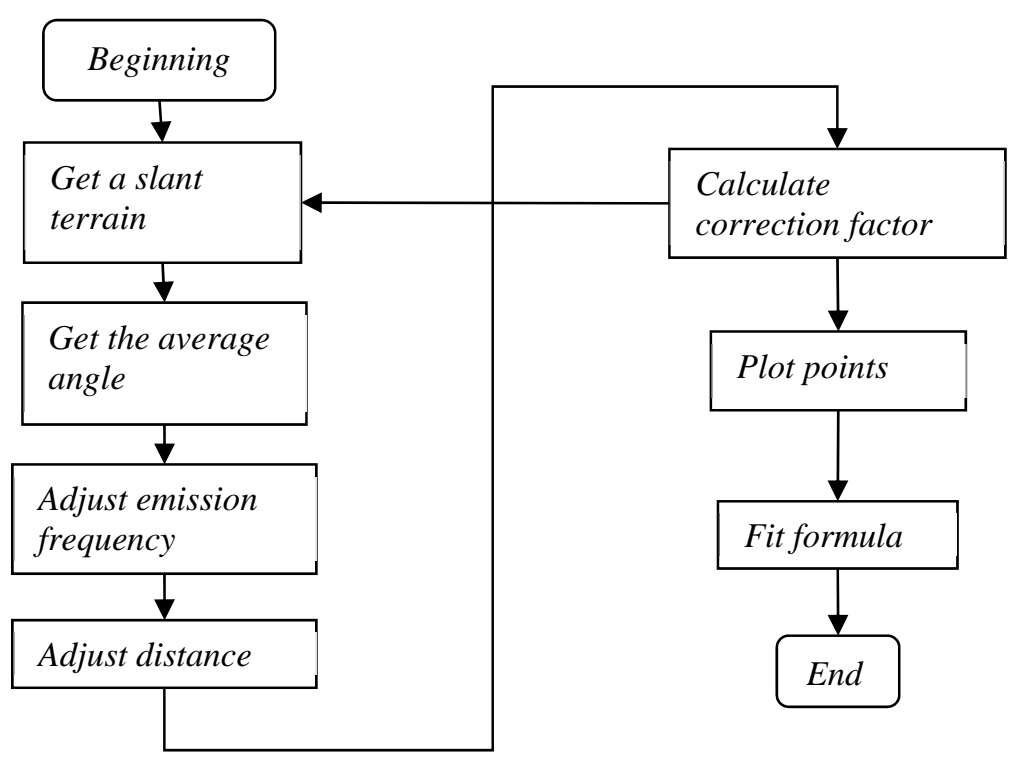

Fig 3. Correction methods for a slant terrain

\section{Summary}

This article firstly contrasts applicability of deterministic models and statistical models in background of tactical wireless communication. Because of the constantly changing radio propagation environment in mobile communication, the statistical models are suitable for calculating propagation loss.

Then, for hilly terrain, slant terrain and edge peaks, the corresponding Okumura correction factors are briefly explained. According to analyze them, such as frequency, it concludes that Okumura correction factors cannot be directly used to correct VHF propagation loss.

Finally, combining with characteristics of Okumura correction factors, the engineering practice process is reworked out from measured data. Those steps can fit correction factors again.

It will get valuable information to VHF communication which is a gap for land terrain correction factors.

\section{References}

[1]. YOUSSEFN.WANGC, PATZOLDM.A study on the second order statistics of mobile fading channels[J]. Vehicular Technology, IEEE Transactions on,2005,54(4):1259-1265.

[2]. CHRYSSOMALLISM. Simulation of mobile fading channels[J]. Antennas and Propagation Magation, IEEE,2002,44(6):172-183.

[3]. PATELC, STUBERG. Efficient simulation of Rayleigh faded cellular channels[J]. Communications, IEEE Transactions on,2005,53(6):1017-1026.

[4]. ZAJICA, STUBERC. Efficient simulation of Rayleigh fading with enhanced de-correlation properties[J]Wireless Communications, IEEE Transaction on,2006,5(7):1866-1875.

[5]. PEDERSENT.FLEURY BH. Modeling of reverberant radio channels suing propagation graphs[J]. IEEE Transactions on Antennas and Propagation,2012,60(12):5978-5988. 